\title{
A MATCHING OF SINGULARITIES IN DOMAIN DECOMPOSITION METHODS FOR REACTION-DIFFUSION PROBLEMS WITH DISCONTINUOUS COEFFICIENTS
}

\author{
Chokri ChNiti ${ }^{1}$
}

\begin{abstract}
In this paper we certify that the same approach proposed in previous works by Chniti et al. [C. R. Acad. Sci. 342 (2006) 883-886; CALCOLO 45 (2008) 111-147; J. Sci. Comput. 38 (2009) 207-228] can be applied to more general operators with strong heterogeneity in the coefficients. We consider here the case of reaction-diffusion problems with piecewise constant coefficients. The problem reduces to determining the coefficients of some transmission conditions to obtain fast convergence of domain decomposition methods. After explaining the theoretical results, we explicitly compute the coefficients in the transmission boundary conditions. The numerical results presented in this paper confirm the optimality properties.
\end{abstract}

Mathematics Subject Classification. 65N55, 35J05, 65N30, 35J25, 35R05.

Received July 1st, 2008. Revised August 6, 2009 and December 21, 2009.

Published online April 15, 2010.

\section{INTRODUCTION}

In this paper we consider an elliptic equation with highly heterogeneous coefficients

$$
\left\{\begin{aligned}
-\nabla \cdot(\nu(x) \nabla u)+\eta(x) u & =f \text { in } \Omega \subset \mathbb{R}^{2}, \\
u & =0 \text { on } \gamma, \\
\frac{\partial u}{\partial n} & =g \text { on } \partial \Omega \backslash \gamma,
\end{aligned}\right.
$$

where $\gamma \subset \partial \Omega$ corresponds to the piece of the boundary with Dirichlet boundary condition. This equation is used for the numerical modelling of the so-called skin problem that describes penetration of drugs through the skin [8]. To simplify the model we choose $\Omega$ as a fragment with one cell with a lipid layer (see Fig. 1). Typical feature for the skin problem is the highly heterogeneous coefficients: the penetration coefficient inside the cells is very small, but it is relatively large in the lipid layer. In this paper we consider this problem with the domain $\Omega$ decomposed into two subdomains, and we restrict our attention to the case where $\gamma=\partial \Omega$. For large numerical computations with these problems, domain decomposition is a natural idea, a non-overlapping decomposition being directly induced by the different materials. We study in this paper the influence of the transmission conditions on the Schwarz algorithm for reaction-diffusion problems. We numerically test improved transmission conditions with second-order tangential derivatives, which were derived from an asymptotic analysis of the Schwarz algorithm

Keywords and phrases. Corner singularity, domain decomposition method, Kondratiev theory.

1 RICAM, Austrian Academy of Sciences, Altenberger strasse 69, 4040 Linz, Austria. chokri.chniti@ricam.oeaw.ac.at 


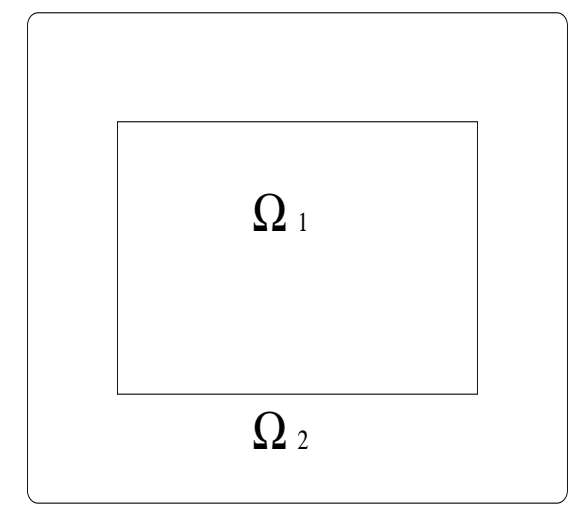

FIGURE 1. A skin fragment consisting of one cell $\left(\Omega_{1}\right)$ and a lipid layer $\left(\Omega_{2}\right): \nu(x)=\nu_{1}$ inside the cell and $\nu(x)=\nu_{2}$ in the lipid layer, with $\nu_{1} \ll \nu_{2}$.

near the corners of the domain. The theoretical optimality of the asymptotic analysis based on the matching of the main singularities within Kondratiev theory [9] is confirmed by numerical computations. The paper is organized as follows. In Section 2, we introduce the model problem and the interface condition used in this paper. In Section 3, a rapid review of some consequences of Kondratiev theory [9] is presented. In Section 4, we recall the strategy of improving the convergence rates around corner singularities and derive an optimal choice of the transmission conditions near the corner. In the remainder of the paper, we give some practical examples and numerical experiments which confirm the optimality of such coefficients.

\section{NeW interface CONDITIONS}

Valid interface conditions in the vicinity of the corners are deduced from regular interfaces conditions (case without corners). We recall some results of the regular cases, and propose new interface conditions valid near the corner.

\subsection{Case of a regular interface}

We consider the reaction-diffusion problems:

$$
\left\{\begin{aligned}
-\nabla \cdot(\nu(x) \nabla u)+\eta(x) u & =f \quad \text { for } x \in \Omega \subset \mathbb{R}^{2} \\
|u| & <+\infty \text { as } x \rightarrow \infty
\end{aligned}\right.
$$

where $f$ is the right-hand side, and the scalar diffusion coefficient $\nu(x)$ and $\eta(x)$ are piecewise constant

$$
\nu(x)=\left\{\begin{array}{l}
\nu_{1} \text { in } \Omega_{1} \\
\nu_{2} \text { in } \Omega_{2}
\end{array} \quad \eta(x)=\left\{\begin{array}{l}
\eta_{1} \text { in } \Omega_{1} \\
\eta_{2} \text { in } \Omega_{2} .
\end{array}\right.\right.
$$

We consider here the model problem posed on the infinite plane $\Omega=\mathbb{R}^{2}$. It can be decomposed into two half-planes, $\Omega_{1}=(-\infty, 0) \times \mathbb{R}$ and $\Omega_{2}=(0,+\infty) \times \mathbb{R}$. The solution $u$ must satisfy two conditions at the interface $x=0$ :

$$
u\left(0^{+}, y\right)=u\left(0^{-}, y\right), \text { and } \quad \nu_{1} \frac{\partial u}{\partial x}\left(0^{+}, y\right)=\nu_{2} \frac{\partial u}{\partial x}\left(0^{-}, y\right), \quad y \in \mathbb{R}
$$


These conditions are simply the continuity of $u$ and of the flux. We wish to analyze the performance of the Schwarz iteration, and minimize the convergence factor over all frequencies relevant to the problem, see [5,10]: if $\left(u_{1}^{n}, u_{2}^{n}\right)$ are known, the step $n+1$ is determined by solving

$$
\begin{aligned}
& \left\{\begin{aligned}
-\nu_{1} \Delta u_{1}^{n+1}+\eta_{1} u_{1}^{n+1} & =f \quad \text { in } \Omega_{1}, \\
\left(\nu_{1} \frac{\partial}{\partial n_{1}}+\mathcal{S}_{2}\right) u_{1}^{n+1}(0, y) & =\left(-\nu_{2} \frac{\partial}{\partial n_{2}}+\mathcal{S}_{2}\right) u_{2}^{n}(0, y), \quad y \in \mathbb{R} \\
\left|u_{1}^{n+1}\right| & <\infty,
\end{aligned}\right. \\
& \left\{\begin{aligned}
-\nu_{2} \Delta u_{2}^{n+1}+\eta_{2} u_{2}^{n+1} & =f \quad \text { in } \Omega_{2}, \\
\left(\nu_{2} \frac{\partial}{\partial n_{2}}+\mathcal{S}_{1}\right) u_{2}^{n+1}(0, y) & =\left(-\nu_{1} \frac{\partial}{\partial n_{1}}+\mathcal{S}_{1}\right) u_{1}^{n}(0, y), \quad y \in \mathbb{R} \\
\left|u_{2}^{n+1}\right| & <\infty,
\end{aligned}\right.
\end{aligned}
$$

where $\mathcal{S}_{j}, j=1,2$, are linear operators acting in the $y$ direction only. By linearity, it will be sufficient to consider only the homogeneous case $f=0$. Our simple model problem allows us to use a Fourier transform in the $y$ variable, i.e.:

$$
\hat{u}(x, k)=\int_{-\infty}^{+\infty} u(x, y) \mathrm{e}^{-\mathrm{i} y k} \mathrm{~d} y,
$$

to analyze the convergence of the Schwarz method (2.2) and (2.3). A natural question is how to choose the transmission conditions in the Schwarz method to get fast convergence "in terms of iterations". Good choices of the transmission conditions optimize the performance of the Schwarz iteration. The main idea is to fix a certain class of local transmission conditions and to optimize the convergence factor of the iteration over this class, for further details see [5]. To do this, we need to have an explicit expression for the convergence factor $\rho$, although it is hard to estimate in general. For the reaction-diffusion problems, the convergence can be fully analyzed, and a convergence factor $\rho(k)$ (as a function of the frequencies tangent to the interface) can be obtained using a Fourier transform. This strategy was first introduced in [7] for the advection-diffusion equation, where a certain subclass of second order conditions was optimized for non-overlapping subdomains. By requiring that the solution in each subdomain be bounded at infinity, and applying the transmission conditions that couple the two subdomains, we find the convergence factor

$$
\rho(k)=\left|\frac{\sigma_{2}(k)-\nu_{2} \sqrt{k^{2}+c_{2}}}{\sigma_{2}(k)+\nu_{1} \sqrt{k^{2}+c_{1}}} \quad \frac{\sigma_{1}(k)-\nu_{1} \sqrt{k^{2}+c_{1}}}{\sigma_{1}(k)+\nu_{2} \sqrt{k^{2}+c_{2}}}\right|,
$$

where $c_{i}=\frac{\eta_{i}}{\nu_{i}}$ and $\sigma_{j}(k)$ are the Fourier symbols of the operators $\mathcal{S}_{j}$. Practically used and efficient interface conditions involve second order tangential derivatives,

$$
\mathcal{S}_{j}=\nu_{j}\left(\beta-\frac{\partial}{\partial \tau}\left(\frac{\alpha}{2} \frac{\partial}{\partial \tau}\right)\right)
$$

where $\frac{\partial}{\partial \tau}$ denotes the tangential derivative and $\alpha$ and $\beta$ are constants. A simple computation gives

$$
\sigma_{j}(k)=\nu_{j}\left(\beta+\frac{\alpha}{2} k^{2}\right)
$$

Let $h$ be the characteristic mesh size of the numerical discretization. Assuming $\Omega$ is split into two half-planes and for a given bounded set of frequencies $\left(k_{1}, k_{2}\right)$ in the tangential variable with $k_{1}>0$ and $k_{2}$ proportional to $\frac{1}{h}$, there is an optimal choice of $(\alpha, \beta)$ for the convergence of the domain decomposition process $(2.2)-(2.3)$. By introducing the error $e_{i}^{n}(x, \tau)=u_{i}^{n}-u$ at step $n$ and its Fourier transform $\widehat{e_{i}^{n}}(x, k)$ in the tangential variable, the optimized coefficients $\alpha_{\text {opt }}>0$ and $\beta_{\text {opt }}>0$ are determined according to [4] by the min-max problem

$$
\min _{\alpha>0, \beta \geq 0}\left(\max _{0<k_{1} \leq k \leq k_{2}}|\rho(k ; \alpha, \beta)|\right),
$$


where $k_{1}$ is a lower bound on the frequency range, $k_{2}$ is a maximal frequency which can be represented on a grid with grid spacing $h$ (an estimate for this maximal frequency is $\frac{\pi}{h}$ ), and the convergence factor is

$$
\rho(k ; \alpha, \beta)=\left|\begin{array}{ll}
\frac{\beta+\frac{\alpha}{2} k^{2}-\sqrt{k^{2}+c_{2}}}{\beta+\frac{\alpha}{2} k^{2}+\frac{1}{\lambda} \sqrt{k^{2}+c_{1}}} & \frac{\beta+\frac{\alpha}{2} k^{2}-\sqrt{k^{2}+c_{1}}}{\beta+\frac{\alpha}{2} k^{2}+\lambda \sqrt{k^{2}+c_{2}}}
\end{array}\right|,
$$

where $\lambda=\frac{\nu_{2}}{\nu_{1}}$.

Theorem 2.1 (cf. [4]). The min-max problem (2.4) has a unique solution given by:

$$
\begin{aligned}
k^{\star} & =\sqrt{\frac{\pi}{\lambda+1}\left(\lambda \sqrt{k_{1}^{2}+c_{1}}+\sqrt{k_{1}^{2}+c_{2}}\right)} h^{-\frac{1}{2}}, \\
\beta_{\mathrm{opt}} & =\left[\frac{\pi}{4(\lambda+1)^{3}}\left(\lambda \sqrt{k_{1}^{2}+c_{1}}+\sqrt{k_{1}^{2}+c_{2}}\right)^{3}\right]^{\frac{1}{4}} h^{-\frac{1}{4}},
\end{aligned}
$$

and

$$
\alpha_{\mathrm{opt}}=2\left[\frac{\lambda+1}{4 \pi^{3}\left(\lambda \sqrt{k_{1}^{2}+c_{1}}+\sqrt{k_{1}^{2}+c_{2}}\right)}\right]^{\frac{1}{4}} h^{\frac{3}{4}} .
$$

After this presentation of some results in the case of a regular interface, our goal now is to give the form of interface conditions available near the corner and their optimal parameters.

\subsection{Interface conditions in the vicinity of the corner}

The decomposition of a regular domain into non-overlapping subdomains can produce interior artificial corners on the interfaces. This makes artificial singularities appear in the solution of the subdomain problems. In order to have a good study of our problem one can consider a domain with only one interior corner. Here the full domain $\Omega=\mathbb{R}^{2}=\mathbb{R}_{+}^{*} \times S^{1} \cup\{O\}$ written in polar coordinates $(r, \theta)$ where the origin $O$ corresponds to $r=0$, will be decomposed into two non-overlapping sectorial subdomains $\bar{\Omega}=\overline{\Omega_{1}} \cup \overline{\Omega_{2}}$, with $\Omega_{1}=\mathbb{R}_{+}^{*} \times\left(\theta_{-}, \theta_{+}\right)$, $\Omega_{2}=\mathbb{R}_{+}^{*} \times\left(\theta_{+}, \theta_{-}+2 \pi\right)$ and $\theta_{+}-\theta_{-} \in(0,2 \pi)$. In the case of a singular domain, following the approach presented in $[1,2]$ (see also the discussion in [11]), the interface condition of the regular interface must be modified in the vicinity of the corner. Indeed, one can show that asymptotically (as $r \rightarrow 0$ ) the interface conditions behave as Dirichlet interface conditions and do not transmit information well from one subdomain to its neighboring ones, for further details see [2]. This was considered in [11] as the explanation of a slow convergence of a domain decomposition algorithm around corners. One way to solve this problem is to force all the terms of the boundary interface operators to have the same homogeneity degree. Thus, a good candidate for the boundary conditions has the form $\pm \nu_{i} \frac{\partial}{r \partial \theta}+\widetilde{\mathcal{S}}_{j}$ where

$$
\widetilde{\mathcal{S}}_{j}=\nu_{j}\left(\frac{\beta_{1,2}}{r}-\frac{\partial}{\partial r}\left(\frac{\alpha_{1,2}}{2} r \frac{\partial}{\partial r}\right)\right)
$$

holds around the corner $r=0$, with $\alpha_{1,2}$ and $\beta_{1,2}$ constant. Far from the corner, the interface boundary must keep the optimal form of the regular interface, for further details see [2]. In summary, we take

$$
\begin{gathered}
B_{i, j}\left( \pm \nu_{i} \frac{\partial}{r \partial \theta} u, u\right)= \pm \nu_{i} \frac{\partial}{r \partial \theta} u+\nu_{j} \widetilde{\beta}_{1,2}(r) u-\nu_{j} \frac{\partial}{\partial r} \frac{\widetilde{\alpha}_{1,2}(r)}{2} \frac{\partial}{\partial r} u, \\
\text { with } \widetilde{\alpha}_{1,2}(r)=\left\{\begin{array}{c}
\alpha_{1,2} r \text { if } r \leq \frac{\alpha_{\mathrm{opt}}}{\alpha_{1,2}}, \\
\alpha_{\mathrm{opt}} \text { if } r \geq \frac{\alpha_{\mathrm{opt}}}{\alpha_{1,2}},
\end{array} \quad \widetilde{\beta}_{1,2}(r)=\left\{\begin{array}{c}
\frac{\beta_{1,2}}{r} \text { if } r \leq \frac{\beta_{1,2}}{\beta_{\mathrm{opt}}}, \\
\beta_{\mathrm{opt}} \text { if } r \geq \frac{\beta_{1,2}}{\beta_{\mathrm{opt}}},
\end{array}\right.\right.
\end{gathered}
$$

where $\alpha_{1,2}>0, \beta_{1,2} \geq 0$ and the pair $\left(\alpha_{\mathrm{opt}}, \beta_{\mathrm{opt}}\right)$ is given by Theorem 2.1. 
In the next sections, we will see how to choose $\alpha_{1}, \beta_{1}$ in (2.6) in order to reduce the corner singularities of $u_{1}^{n}$ (the same can be done for $u_{2}^{n}$ ), which is a solution of

$$
\left\{\begin{aligned}
-\nu_{1} \frac{1}{r^{2}}\left(\left(r \partial_{r}\right)^{2}+\partial_{\theta}^{2}\right) u_{1}^{n+1}(r, \theta)+\eta_{1} u_{1}^{n+1}(r, \theta) & =0 \\
\left(-\nu_{1} \frac{1}{r} \partial_{\theta}+\widetilde{\mathcal{S}}_{2}\right) u_{1}^{n+1}\left(r, \theta_{-}\right) & =\left(-\nu_{2} \frac{1}{r} \partial_{\theta}+\widetilde{\mathcal{S}}_{2}\right) u_{2}^{n}\left(r, \theta_{-}+2 \pi\right), \\
\left(\nu_{1} \frac{1}{r} \partial_{\theta}+\widetilde{\mathcal{S}}_{2}\right) u_{1}^{n+1}\left(r, \theta_{+}\right) & =\left(\nu_{2} \frac{1}{r} \partial_{\theta}+\widetilde{\mathcal{S}}_{2}\right) u_{2}^{n}\left(r, \theta_{+}\right) .
\end{aligned}\right.
$$

\section{Corner Singularities ANALysis}

An important ingredient for the analysis of corner singularities is the Mellin transform (see [2] for further details) with respect to the radial variable, defined as

$$
\widehat{f}(z)=\mathcal{M}(f)(z)=\int_{0}^{\infty} r^{i z} f(r) \frac{\mathrm{d} r}{r} .
$$

Recall that

$$
\mathcal{M}\left(\operatorname{ir} \partial_{r} f\right)(z)=z \mathcal{M}(f)(z)
$$

Then the asymptotic expansion of $u$, with $\operatorname{supp} u \subset\{r \leq 1\}$ :

$$
u(r, \theta)=\sum_{k=1}^{\infty} \sum_{j=0}^{\mu_{k}-1} a_{k, j} r^{-i z_{k}} \ln ^{j}(r) \varphi_{k, j}(\theta)
$$

can be written in terms of $\mathcal{M} u$ which is meromorphic in some upper half-complex plane by noticing that

- $z_{k}$ are the poles of $\mathcal{M} u$;

- $\mu_{k}$ are their corresponding multiplicity;

- $\varphi_{k, j}$ encode the angular variations;

- $a_{k, j} \in \mathbb{R}$.

When one solves the reaction-diffusion problems with Dirichlet boundary condition in some domain $\Omega=\left\{(r, \theta), 0<r<1, \theta_{-}<\theta<\theta_{+}\right\}$for real values $\theta_{ \pm} \in(0,2 \pi)$ and with a vanishing $f$ around $\{r=0\}$, the main term of the asymptotic expansion is obtained by looking for the $z$ 's for which the angular boundary value problem

$$
\left\{\begin{aligned}
\left(\partial_{\theta}\left(\nu \partial_{\theta}\right)-\nu z^{2}\right) \hat{e}(z, \theta) & =0, \\
\hat{e}\left(z, \theta_{-}\right) & =0, \\
\hat{e}\left(z, \theta_{+}\right) & =0,
\end{aligned}\right.
$$

admits a non-trivial solution. The $z^{2}$ are actually the poles of the meromorphic resolvent attached to the asymptotic as $r \rightarrow 0$ angular problem according to Kondratiev theory, see [9] for further details.

\subsection{Expansion of a solution to the problem in $\Omega$}

The final aim of our work is to improve the convergence of domain decomposition methods in the neighborhood of corners. The relevant analysis has to be done in a neighborhood of $r=0$. It is sufficient to consider truncated solutions $\psi(r) u$, with $\psi \in \mathcal{C}_{0}^{\infty}\left(\mathbb{R}_{+}\right)$, supp $\psi \subset\{r \leq 1\}$ and $\psi \equiv 1$ in a neighborhood of $\{r=0\}$. One can even consider directly the the situation $u=\psi(r) u$. The natural singularities exponent and the angular functions associated with the boundary value problem (1.1) are given by the next proposition.

Proposition 3.1. Let $\Theta=\theta_{+}-\theta_{-}-\pi$, and $\kappa=\left(\frac{\nu_{2}-\nu_{1}}{\nu_{2}+\nu_{1}}\right)^{2}$. The complex numbers $z$ for which (1.1) admits a non-trivial solution are given by $z=i t, t \in \mathbb{R}$ with

$$
\sin ^{2}(\pi t)=\kappa \sin ^{2}(\Theta t) .
$$


Proof. We note that $e=\psi e$, where $\psi$ is a smooth function with compact support and $e$ is the solution of the homogeneous equation associated to the principal part of the operator. Considering the principal part as $r \rightarrow 0$ of the problem (1.1) and applying the Mellin transform, we are led to consider the equation:

$$
\left(\partial_{\theta}\left(\nu \partial_{\theta}\right)-\nu z^{2}\right) \hat{e}(z, \theta)=0 .
$$

Here $\hat{e}(z,$.$) is an eigenfunction of the operator K(\theta)=-\frac{\partial}{\partial \theta}\left(\nu \frac{\partial}{\partial \theta}\right)$, defined on

$$
\mathcal{D}(\theta)=\left\{u \in H^{1}(\Omega),\left[\nu \frac{\partial u}{\partial \theta}\right]_{\mid \partial \Omega_{1} \cap \partial \Omega_{2}}=0\right\}
$$

for the eigenvalue $-\nu z^{2}$. Moreover, $K(\theta)$ is a self-adjoint positive operator and thus $z^{2} \leq 0$ and $z=i t$ with $t>0$. As $\nu(x)$ is a piecewise constant coefficient, we solve the problem in each subdomain. A simple calculation gives

$$
A_{i} \cos (t \theta)+B_{i} \sin (t \theta) \text { in } \Omega_{i}
$$

Moreover, we impose that on the interface $\left\{\theta=\theta_{ \pm}\right\}$we have

$$
\begin{aligned}
\left.\hat{e}(t, \theta)\right|_{\Omega_{1}, \theta_{+}} & =\left.\hat{e}(t, \theta)\right|_{\Omega_{2}, \theta_{+}} \\
\left.\hat{e}(t, \theta)\right|_{\Omega_{1}, \theta_{-}} & =\left.\hat{e}(t, \theta)\right|_{\Omega_{2}, \theta_{-}+2 \pi} \\
\left.\nu_{1} \frac{\partial}{\partial \theta} \hat{e}(t, \theta)\right|_{\Omega_{1}, \theta_{+}} & =\left.\nu_{2} \frac{\partial}{\partial \theta} \hat{e}(t, \theta)\right|_{\Omega_{2}, \theta_{+}} \\
\left.\nu_{1} \frac{\partial}{\partial \theta} \hat{e}(t, \theta)\right|_{\Omega_{1}, \theta_{-}} & =\left.\nu_{2} \frac{\partial}{\partial \theta} \hat{e}(t, \theta)\right|_{\Omega_{2}, \theta_{-}+2 \pi}
\end{aligned} .
$$

This leads to

$$
M_{\nu_{1}, \nu_{2}}(t)\left(\begin{array}{c}
A_{1} \\
B_{1} \\
A_{2} \\
B_{2}
\end{array}\right)=0_{\mathbb{R}^{4}}
$$

where

$$
M_{\nu_{1}, \nu_{2}}(z)=\left(\begin{array}{cccc}
\cos \left(t \theta_{+}\right) & \sin \left(t \theta_{+}\right) & -\cos \left(t \theta_{+}\right) & -\sin \left(t \theta_{+}\right) \\
\cos \left(t \theta_{-}\right) & \sin \left(t \theta_{-}\right) & -\cos \left(t\left(\theta_{-}+2 \pi\right)\right) & -\sin \left(t\left(\theta_{-}+2 \pi\right)\right) \\
-\nu_{1} \sin \left(t \theta_{+}\right) & \nu_{1} \cos \left(t \theta_{+}\right) & \nu_{2} \sin \left(t \theta_{+}\right) & -\nu_{2} \cos \left(t \theta_{+}\right) \\
-\nu_{1} \sin \left(t \theta_{-}\right) & \nu_{1} \cos \left(t \theta_{-}\right) & \nu_{2} \sin \left(t\left(\theta_{-}+2 \pi\right)\right) & -\nu_{2} \cos \left(t\left(\theta_{-}+2 \pi\right)\right)
\end{array}\right) .
$$

The poles are solutions of $\operatorname{det} M_{\nu_{1}, \nu_{2}}(t)=0$. A straightforward computation then gives

$$
\frac{\left(\nu_{1}+\nu_{2}\right)^{2}}{4 \nu_{1} \nu_{2}} \cos (2 \pi t)-\frac{\left(\nu_{1}-\nu_{2}\right)^{2}}{4 \nu_{1} \nu_{2}} \cos \left(2 t\left(\theta_{+}-\theta_{-}-\pi\right)\right)=1 .
$$

Therefore, the poles are solutions of

$$
\sin ^{2}(\pi t)=\kappa \sin ^{2}\left(t\left(\theta_{+}-\theta_{-}-\pi\right)\right),
$$

where $\kappa=\left(\frac{\nu_{2}-\nu_{1}}{\nu_{2}+\nu_{1}}\right)^{2}$.

Then, the first term in the asymptotic expansion of $u$ is given by

$$
e(r, \theta)=A_{0}+r^{\tau_{1}}\left\{\begin{array}{ll}
A_{1} \cos \left(\tau_{1} \theta\right)+B_{1} \sin \left(\tau_{1} \theta\right) & \text { in } \Omega_{1} \\
A_{2} \cos \left(\tau_{1} \theta\right)+B_{2} \sin \left(\tau_{1} \theta\right) & \text { in } \Omega_{2}
\end{array}+o\left(r^{\tau_{1}}\right),\right.
$$

where $\tau_{1}$ denotes the first positive solution of (3.3). 


\subsection{Subproblem in $\boldsymbol{\Omega}_{\mathbf{1}}$}

We focus on the subdomain $\Omega_{1}$, the treatment of $\Omega_{2}$ being similar. The boundary problem with the selected interface conditions solved with the error $e_{1}^{n+1}=u_{1}^{n+1}-u$ reads

$$
\left\{\begin{aligned}
-\nu_{1} \frac{1}{r^{2}}\left(\left(r \partial_{r}\right)^{2}+\partial_{\theta}^{2}\right) e_{1}^{n+1}(r, \theta)+\eta_{1} e_{1}^{n+1}(r, \theta) & =0 \\
\left(-\nu_{1} \frac{1}{r} \partial_{\theta}+\widetilde{\mathcal{S}}_{2}\right) e_{1}^{n+1}\left(r, \theta_{-}\right) & =\left(-\nu_{2} \frac{1}{r} \partial_{\theta}+\widetilde{\mathcal{S}}_{2}\right) e_{2}^{n}\left(r, \theta_{-}+2 \pi\right), \\
\left(\nu_{1} \frac{1}{r} \partial_{\theta}+\widetilde{\mathcal{S}}_{2}\right) e_{1}^{n+1}\left(r, \theta_{+}\right) & =\left(\nu_{2} \frac{1}{r} \partial_{\theta}+\widetilde{\mathcal{S}}_{2}\right) e_{2}^{n}\left(r, \theta_{+}\right),
\end{aligned}\right.
$$

where

$$
\widetilde{\mathcal{S}}_{2}=\nu_{2}\left(\frac{\beta_{1}}{r}-\frac{\partial}{\partial r}\left(\frac{\alpha_{1}}{2} r \frac{\partial}{\partial r}\right)\right) .
$$

The case $\beta \neq 0$ is not permitted in interior corners of $\Omega=\mathbb{R}^{2}$, or more generally when the general solution of the complete problem does not vanish at $r=0$ (for further details see [2]). The main singularities associated with this problem are derived following Kondratiev theory [9]. By considering the principal part as $r \rightarrow 0$ and by applying the Mellin transform, leads to the system

$$
\left\{\begin{aligned}
\left(\partial_{\theta}^{2}-z^{2}\right) \widehat{e_{1}^{n+1}}(z, \theta) & =0 \\
\left(\partial_{\theta}-\lambda \frac{\alpha_{1}}{2} z^{2}\right) \widehat{e_{1}^{n+1}}\left(z, \theta_{-}\right) & =\widehat{g_{-}^{n}}(z), \\
\left(\partial_{\theta}+\lambda \frac{\alpha_{1}}{2} z^{2}\right) \widehat{e_{1}^{n+1}}\left(z, \theta_{+}\right) & =\widehat{g_{+}^{n}}(z),
\end{aligned}\right.
$$

where

$$
\widehat{g_{+}^{n}}(z)=\lambda\left(\partial_{\theta}+\frac{\alpha_{1}}{2} z^{2}\right) \widehat{e_{2}^{n}}\left(z, \theta_{+}\right), \quad \widehat{g_{-}^{n}}(z)=\lambda\left(\partial_{\theta}-\frac{\alpha_{1}}{2} z^{2}\right) \widehat{e_{2}^{n}}\left(z, \theta_{-}+2 \pi\right), \quad \lambda=\frac{\nu_{2}}{\nu_{1}},
$$

and whose solution is

$$
\begin{aligned}
\widehat{e_{1}^{n+1}} & =a(z) \mathrm{e}^{z\left(\theta-\theta_{-}\right)}+b(z) \mathrm{e}^{-z\left(\theta-\theta_{+}\right)} \\
\left(\begin{array}{l}
a(z) \\
b(z)
\end{array}\right) & =\frac{1}{z} \mathcal{R}(z)\left(\begin{array}{c}
\widehat{g_{+}^{n}}(z) \\
g_{-}^{n}(z)
\end{array}\right) \\
\text { with } \mathcal{R}(z) & =\left(\begin{array}{cc}
\left(1+\lambda \frac{\alpha_{1}}{2} z\right) \mathrm{e}^{z\left(\theta_{+}-\theta_{-}\right)} & -1+\lambda \frac{\alpha_{1}}{2} z \\
1-\lambda \frac{\alpha_{1}}{2} z & -\left(1+\lambda \frac{\alpha_{1}}{2} z\right) \mathrm{e}^{z\left(\theta_{+}-\theta_{-}\right)}
\end{array}\right)^{-1} .
\end{aligned}
$$

Proposition 3.2. The poles with a positive imaginary part of the factor $\mathcal{R}(z)$ are $z=$ it, with

$$
\begin{aligned}
\tan \left(\frac{\pi x t}{2}\right) & =\frac{2}{\lambda \alpha_{1} t} \\
\tan \left(\frac{\pi x t}{2}\right) & =-\frac{\lambda \alpha_{1}}{2} t,
\end{aligned}
$$

with $x=\frac{\theta_{+}-\theta_{-}}{\pi}$, whose positive solutions are denoted by $t_{k}, k \in \mathbb{N}^{*}$, in the increasing order.

Proof. See [2].

It follows immediately from the positiveness of $\lambda=\frac{\nu_{2}}{\nu_{1}}, x$ and $\alpha_{1}$ that the first pole $i t_{1}$ is associated with the equation

$$
\tan \left(\frac{\pi x t}{2}\right)=\frac{2}{\lambda \alpha_{1} t} \text {. }
$$


In the next section, we derive an optimal parameter $\alpha_{1}$ for the subproblem in $\Omega_{1}$ (if it is possible) such that the first pole $i t_{1}$ will be canceled.

\section{Theoretical Choice of the parameter $\alpha_{1}$}

We give here a short summary of the strategy of optimization - for further details refer to [2]. Expecting that better matching yields faster convergence, our strategy to determine the "best parameter" $\left(\alpha_{i}\right)$ is the following: assuming that at step $n$ the error functions $e_{i}^{n}=u_{i}^{n}-u, i=1,2$, have the asymptotic type of the problem on the full domain $\Omega$, the error functions $e_{i}^{n+1}$ should keep this asymptotic type up to some large enough order as $r \rightarrow 0$. (That is, domain decomposition is used for the solution procedure, and for the corresponding iteration we try to define the transmission conditions in such a way that additional artificial singularities in the corner either are suppressed or are at most of the same order as the singularity of the original problem.) The analysis focuses on the first artificial pole, which is the one with the smallest imaginary part. The corresponding residual depends on the right-hand side in (2.7), which for the first order is given by the asymptotic type of the full domain problem. The question now becomes whether it is possible to choose the parameter $\alpha_{i}$ in $\Omega_{i}, i=1,2$, so that if the data involved in the right-hand side of (2.7) has the right asymptotic type, then the first artificial pole is canceled by a vanishing residue. The computation will show that such a choice of the parameter is not always possible, and in the general case we have to choose between two strategies in each subdomain $\Omega_{i}$, $i=1,2$ :

(1) Check if it is possible to cancel the first artificial pole according to the previous process;

(2) If the first approach has no solution, choose the parameter $\alpha_{i}, i=1,2$, in such a way that the first artificial pole has the largest possible imaginary part.

Keep in mind that $\beta_{i}=0$ is necessary ${ }^{2}$ because the solution $u$, has in general, a nonzero value at $r=0$. Only the coefficients $\alpha_{i}$ can be used. In the subdomain $\Omega_{1}$ and for a general right-hand side in (2.7), the first artificial term in the asymptotic expansion of $e_{1}^{n+1}$ appears with the factor $r^{t_{1}}$, with $t_{1}$ defined in Proposition 3.2. The first (and most efficient) approach assumes that at step $n$ the error has the natural asymptotic type associated with the global problem:

$$
e_{2}^{n}(r, \theta)=A_{0}+r^{\tau_{1}}\left(A_{2} \cos \left(\tau_{1} \theta\right)+B_{2} \sin \left(\tau_{1} \theta\right)\right)+o\left(r^{\tau_{1}}\right)
$$

where $\tau_{1}$ denotes the first positive solution of (3.3). With an additional truncation in $\{r \leq R\}$ we set the following as in the domain decomposition algorithm

$$
\begin{aligned}
& g_{+}^{n}(r)=1_{\{r \leq R\}} \lambda\left(\partial_{\theta}-\frac{\alpha_{1}}{2}\left(r \partial_{r}\right)^{2}\right) e_{2}^{n}\left(r, \theta_{+}\right), \\
& g_{-}^{n}(r)=1_{\{r \leq R\}} \lambda\left(\partial_{\theta}+\frac{\alpha_{1}}{2}\left(r \partial_{r}\right)^{2}\right) e_{2}^{n}\left(r, \theta_{-}+2 \pi\right),
\end{aligned}
$$

where $R \in \mathbb{R}_{+}^{*}$. After working with the first order expansion of the global problem, neglecting the $o\left(r^{\tau_{1}}\right)$ remainder and taking the Mellin transform, this provides

$$
\begin{aligned}
\widehat{g_{+}^{n}}(z)= & \frac{R^{\tau_{1}+i z}}{\tau_{1}+i z} \lambda \tau_{1}\left\{A_{2}\left(-\sin \left(\tau_{1} \theta_{+}\right)-\tau_{1} \frac{\alpha_{1}}{2} \cos \left(\tau_{1} \theta_{+}\right)\right)+B_{2}\left(\cos \left(\tau_{1} \theta_{+}\right)-\tau_{1} \frac{\alpha_{1}}{2} \sin \left(\tau_{1} \theta_{+}\right)\right)\right\} \\
\widehat{g_{-}^{n}}(z)= & \frac{R^{\tau_{1}+i z}}{\tau_{1}+i z} \lambda \tau_{1}\left\{A_{2}\left(-\sin \left(\tau_{1}\left(\theta_{-}+2 \pi\right)\right)+\tau_{1} \frac{\alpha_{1}}{2} \cos \left(\tau_{1}\left(\theta_{-}+2 \pi\right)\right)\right)\right. \\
& \left.+B_{2}\left(\cos \left(\tau_{1}\left(\theta_{-}+2 \pi\right)\right)+\tau_{1} \frac{\alpha_{1}}{2} \sin \left(\tau_{1}\left(\theta_{-}+2 \pi\right)\right)\right)\right\} .
\end{aligned}
$$

We note that the boundary conditions do not contain a constant term $\left(\partial_{\theta}-\frac{\alpha_{1}}{2}\left(r \partial_{r}\right)^{2}\right)$ and therefore $g_{ \pm}^{n}$ do not depend on the first term $\left(A_{0}\right)$ in the expansion of $e_{2}^{n}$.

\footnotetext{
${ }^{2}$ Practically, the case $\beta_{i}=0$ is implemented by keeping a constant coefficient $\tilde{\beta}(r)=\beta_{\text {opt }}$ along the whole interface.
} 
Our goal is to see whether there exists $\alpha_{1}$ such that

$$
\mathcal{R}(z)\left(\begin{array}{l}
\widehat{g_{+}^{n}}(z) \\
\widehat{g_{-}^{n}}(z)
\end{array}\right) \text { does not have any more pole on } i t_{1}\left(t_{1}>0\right) .
$$

According to $[1,2]$, here the cancellation of the first artificial pole $i t_{1}$ is reduced to the simple condition

$$
\widehat{g_{+}^{n}}\left(i t_{1}\right)=-\widehat{g_{-}^{n}}\left(i t_{1}\right) \text {. }
$$

Proposition 4.1. The equation (4.1) gives

$$
\alpha_{1}=\frac{2}{\tau_{1}} \frac{1}{\tan \left(\frac{\theta_{+}-\theta_{-}-2 \pi}{2} \tau_{1}\right)}
$$

for $\frac{\theta_{+}-\theta_{-}}{\pi} \in\left(2-\frac{2}{\tau_{1}}, 2-\frac{1}{\tau_{1}}\right)$.

Proof. Equation (4.1) does not depend on the truncation parameter $R$ and reads simply

$$
\alpha_{1}=\alpha_{1}=\frac{2}{\tau_{1}} \frac{1}{\tan \left(\frac{\theta_{+}-\theta_{-}-2 \pi}{2} \tau_{1}\right)} .
$$

We recall that $0<\theta_{+}-\theta_{-}<2 \pi$, which implies

$$
-\pi<\frac{\theta_{+}-\theta_{-}-2 \pi}{2} \tau_{1}<0
$$

If $\frac{\theta_{+}-\theta_{-}-2 \pi}{2} \tau_{1} \in\left(-\pi,-\frac{\pi}{2}\right)$, then the parameter $\alpha_{1}$ is positive and this yields the result.

Remark 4.2. If $\nu_{1}=\nu_{2}$, condition (4.2) becomes

$$
\alpha_{1}=\frac{2}{\tan \left(\frac{\theta_{+}-\theta_{-}}{2}\right)} \text { for } \frac{\theta_{+}-\theta_{-}}{\pi} \in(0,1)
$$

and thus, we recover the optimized coefficients of the Laplace operator in the same domain [1-3].

\section{EXAMPLES}

The characteristic equation (3.3) cannot be solved explicitly. Therefore, in this section we consider the case $\theta_{+}-\theta_{-}=\frac{\pi}{2}$. In this case we can explicitly compute the first pole

$$
\tau_{1}=\frac{1}{\pi} \arccos \left(-\frac{\nu_{1}^{2}+\nu_{2}^{2}+6 \nu_{1} \nu_{2}}{2\left(\nu_{1}+\nu_{2}\right)^{2}}\right) .
$$

Moreover, $\tau_{1} \in\left(\frac{2}{3}, 1\right)$. Then $(3.3)$ yields

$$
\alpha_{1}=-\frac{2}{\tau_{1}} \frac{1}{\tan \left(\frac{3 \pi}{4} \tau_{1}\right)}>0
$$

In the case when $\theta_{+}-\theta_{-}=\frac{3 \pi}{2}$, the first pole is the same as the case where $\theta_{+}-\theta_{-}=\frac{\pi}{2}$. But, in this case (3.3) gives

$$
\alpha_{1}=-\frac{2}{\tau_{1}} \frac{1}{\tan \left(\frac{\pi}{4} \tau_{1}\right)}<0 .
$$


Therefore, for a given $\theta_{+}-\theta_{-}$we cannot always cancel the first artificial pole, so the two strategies of optimization can be used for our problem. For instance, when the domain $\Omega$ is decomposed into one cell $\Omega_{1}$ and a lipid layer $\Omega_{2}$, correspond to the case of Figure 1, we see that the first strategy is used only in the cell and the second strategy in $\Omega_{2}$. Up to now, we keep in mind that the first approach depending on the angle of the subdomain $\Omega_{i}$, does not admit a solution in all cases. The second approach will also be tested when necessary.

If we consider the case where $\nu_{1}=1$ and $\nu_{2} \gg \nu_{1}$, then the first pole is given by

$$
\tau_{1}=\frac{1}{\pi} \arccos \left(-\frac{1+\nu_{2}^{2}+6 \nu_{2}}{2\left(1+\nu_{2}\right)^{2}}\right) \text {. }
$$

According to the formula for $\tau_{1}$, one sees that $\tau_{1}$ is monotonically decreasing with $\nu_{2}$ (we consider $\nu_{2}$ as parameter) to the value $\frac{2}{3}$. Therefore, the solution of the interface problem belongs to $H^{1+\frac{2}{3}}(\Omega)$ independently of the jump discontinuity of the diffusion coefficient. We recall that the Sobolev space $H^{s}(\Omega), s>0, s \notin \mathbb{N}$, is defined as the space of all distributions with finite norm:

$$
\|v\|_{H^{s}(\Omega)}^{2}=\|v\|_{H^{m}(\Omega)}^{2}+\sum_{|\zeta|=m} \int_{\Omega} \int_{\Omega} \frac{\left|D^{\zeta} v(x)-D^{\zeta} v(y)\right|^{2}}{|x-y|^{2+2 \sigma}} \mathrm{d} x \mathrm{~d} y
$$

where $s=m+\sigma, m \in \mathbb{N}, \sigma \in(0,1)$ and $D^{\zeta}$ denotes the derivatives with respect to the multi-index $\zeta=\left(\zeta_{1}, \zeta_{2}\right)$ (see [6] for more details).

\section{Discrete PROBlem}

Owing to a reformulation of the algorithm the computation of the normal derivative involved in these interface conditions is avoided. For the sake of simplicity we still consider a case with two subdomains, but this procedure can be adapted to any general decomposition with more than two subdomains. We restrict our attention to the additive Schwarz iteration

$$
\begin{aligned}
& \left\{\begin{aligned}
-\nu_{1} \Delta u_{1}^{n+1}+\eta_{1} u_{1}^{n+1} & =f, \\
\left(-\nu_{1} \frac{1}{r} \partial_{\theta}+\widetilde{\mathcal{S}}_{2}\right) u_{1}^{n+1}\left(r, \theta_{-}\right) & =\left(-\nu_{2} \frac{1}{r} \partial_{\theta}+\widetilde{\mathcal{S}}_{2}\right) u_{2}^{n}\left(r, \theta_{-}\right), \\
\left(\nu_{1} \frac{1}{r} \partial_{\theta}+\widetilde{\mathcal{S}}_{2}\right) u_{1}^{n+1}\left(r, \theta_{+}\right) & =\left(\nu_{2} \frac{1}{r} \partial_{\theta}+\widetilde{\mathcal{S}}_{2}\right) u_{2}^{n}\left(r, \theta_{+}\right),
\end{aligned}\right. \\
& \left\{\begin{aligned}
-\nu_{2} \Delta u_{2}^{n+1}+\eta_{2} u_{2}^{n+1} & =f, \\
\left(\nu_{2} \frac{1}{r} \partial_{\theta}+\widetilde{\mathcal{S}}_{1}\right) u_{2}^{n+1}\left(r, \theta_{-}\right) & =\left(\nu_{1} \frac{1}{r} \partial_{\theta}+\widetilde{\mathcal{S}}_{1}\right) u_{1}^{n}\left(r, \theta_{-}\right), \\
\left(-\nu_{2} \frac{1}{r} \partial_{\theta}+\widetilde{\mathcal{S}}_{1}\right) u_{2}^{n+1}\left(r, \theta_{+}\right) & =\left(-\nu_{1} \frac{1}{r} \partial_{\theta}+\widetilde{\mathcal{S}}_{1}\right) u_{1}^{n}\left(r, \theta_{+}\right) .
\end{aligned}\right.
\end{aligned}
$$

A direct discretization would require the computation of the normal derivatives along the interfaces $\left\{\theta=\theta_{ \pm}\right\}$ in order to evaluate the right hand sides in the transmission conditions of (6.2)-(6.1). This can be avoided by introducing four new variables,

$$
\begin{aligned}
& \lambda_{\left(1, \theta_{-}\right)}^{n}=\left(-\nu_{2} \frac{1}{r} \partial_{\theta}+\widetilde{\mathcal{S}}_{2}\right) u_{2}^{n}\left(., \theta_{-}\right), \\
& \lambda_{\left(1, \theta_{+}\right)}^{n}=\left(\nu_{2} \frac{1}{r} \partial_{\theta}+\widetilde{\mathcal{S}}_{2}\right) u_{2}^{n}\left(., \theta_{+}\right), \\
& \lambda_{\left(2, \theta_{-}\right)}^{n}=\left(\nu_{1} \frac{1}{r} \partial_{\theta}+\widetilde{\mathcal{S}}_{1}\right) u_{1}^{n}\left(., \theta_{-}\right), \\
& \lambda_{\left(2, \theta_{+}\right)}^{n}=\left(-\nu_{1} \frac{1}{r} \partial_{\theta}+\widetilde{\mathcal{S}}_{1}\right) u_{1}^{n}\left(., \theta_{+}\right) .
\end{aligned}
$$


The algorithm then becomes

$$
\left\{\begin{aligned}
-\nu_{1} \Delta u_{1}^{n+1}+\eta_{1} u_{1}^{n+1} & =f, \\
\left(-\nu_{1} \frac{1}{r} \partial_{\theta}+\widetilde{\mathcal{S}}_{2}\right) u_{1}^{n+1}\left(r, \theta_{-}\right) & =\lambda_{\left(1, \theta_{-}\right)}^{n}(r), \\
\left(\nu_{1} \frac{1}{r} \partial_{\theta}+\widetilde{\mathcal{S}}_{2}\right) u_{1}^{n+1}\left(r, \theta_{+}\right) & =\lambda_{\left(1, \theta_{+}\right)}^{n}(r), \\
-\nu_{2} \Delta u_{2}^{n+1}+\eta_{2} u_{2}^{n+1} & =f, \\
\left(\nu_{2} \frac{1}{r} \partial_{\theta}+\widetilde{\mathcal{S}}_{1}\right) u_{2}^{n+1}\left(r, \theta_{-}\right) & =\lambda_{\left(2, \theta_{-}\right)}^{n}(r), \\
\left(-\nu_{2} \frac{1}{r} \partial_{\theta}+\widetilde{\mathcal{S}}_{1}\right) u_{2}^{n+1}\left(r, \theta_{+}\right) & =\lambda_{\left(2, \theta_{+}\right)}^{n}(r),
\end{aligned}\right.
$$

and

$$
\begin{aligned}
& \lambda_{\left(1, \theta_{-}\right)}^{n+1}=-\lambda_{\left(2, \theta_{-}\right)}^{n}+\left(\nu_{2} \tilde{\beta}_{1}(r)+\nu_{1} \tilde{\beta}_{2}(r)-\partial_{r}\left(\frac{\left[\nu_{2} \tilde{\alpha}_{1}(r)+\nu_{1} \tilde{\alpha}_{2}(r)\right]}{2} \partial_{r}\right)\right) u_{2}^{n}\left(., \theta_{-}\right), \\
& \lambda_{\left(2, \theta_{-}\right)}^{n+1}=-\lambda_{\left(1, \theta_{-}\right)}^{n}+\left(\nu_{2} \tilde{\beta}_{1}(r)+\nu_{1} \tilde{\beta}_{2}(r)-\partial_{r}\left(\frac{\left[\nu_{2} \tilde{\alpha}_{1}(r)+\nu_{1} \tilde{\alpha}_{2}(r)\right]}{2} \partial_{r}\right)\right) u_{1}^{n}\left(r, \theta_{-}\right), \\
& \lambda_{\left(1, \theta_{+}\right)}^{n+1}=-\lambda_{\left(2, \theta_{+}\right)}^{n}+\left(\nu_{2} \tilde{\beta}_{1}(r)+\nu_{1} \tilde{\beta}_{2}(r)-\partial_{r}\left(\frac{\left[\nu_{2} \tilde{\alpha}_{1}(r)+\nu_{1} \tilde{\alpha}_{2}(r)\right]}{2} \partial_{r}\right)\right) u_{2}^{n}\left(., \theta_{+}\right), \\
& \lambda_{\left(2, \theta_{+}\right)}^{n+1}=-\lambda_{\left(1, \theta_{+}\right)}^{n}+\left(\nu_{2} \tilde{\beta}_{1}(r)+\nu_{1} \tilde{\beta}_{2}(r)-\partial_{r}\left(\frac{\left[\nu_{2} \tilde{\alpha}_{1}(r)+\nu_{1} \tilde{\alpha}_{2}(r)\right]}{2} \partial_{r}\right)\right) u_{1}^{n}\left(., \theta_{+}\right) .
\end{aligned}
$$

The variational formulation of the problem for each subdomain with interface conditions (2.5) is given by

$$
\begin{aligned}
\int_{\Omega_{i}} \eta_{i} u_{i}^{n+1} v_{i}+\int_{\Omega_{i}} \nu_{i} \nabla u_{i}^{n+1} \nabla v_{i}+\int_{0}^{\infty} \nu_{j} \tilde{\beta}_{i}(r)\left(u_{i}^{n+1} v_{i}\right)\left(r, \theta_{ \pm}\right)+\int_{0}^{\infty} \frac{\nu_{j} \tilde{\alpha}_{i}(r)}{2}\left(\partial_{r} u_{i}^{n+1} \partial_{r} v_{i}\right)\left(r, \theta_{ \pm}\right) & =\int_{\Omega_{i}} f v_{i}+\int_{0}^{\infty} \lambda_{\left(i, \theta_{ \pm}\right)}^{n} v_{i}\left(r, \theta_{ \pm}\right)
\end{aligned}
$$

This leads to a matrix form of the algorithm:

$$
\begin{aligned}
\widetilde{\mathcal{K}_{1}} u_{1}^{n+1} & =f+B_{1}^{T} \lambda_{1}^{n}, \\
\widetilde{\mathcal{K}_{2}} u_{2}^{n+1} & =f+B_{2}^{T} \lambda_{2}^{n}, \\
\mathcal{M}_{\Gamma} \lambda_{1}^{n+1} & =-\mathcal{M}_{\Gamma} \lambda_{2}^{n}+\left(\mathcal{M}_{\tilde{\beta}_{1}, \Gamma}+\mathcal{M}_{\tilde{\beta}_{2}, \Gamma}+\mathcal{K}_{\tilde{\alpha}_{1}, \Gamma}+\mathcal{K}_{\tilde{\alpha}_{2}, \Gamma}\right) B_{2} u_{2}^{n+1}, \\
\mathcal{M}_{\Gamma} \lambda_{2}^{n+1} & =-\mathcal{M}_{\Gamma} \lambda_{1}^{n}+\left(\mathcal{M}_{\tilde{\beta}_{1}, \Gamma}+\mathcal{M}_{\tilde{\beta}_{2}, \Gamma}+\mathcal{K}_{\tilde{\alpha}_{1}, \Gamma}+\mathcal{K}_{\tilde{\alpha}_{2}, \Gamma}\right) B_{1} u_{1}^{n+1},
\end{aligned}
$$

where $\lambda_{1}, \lambda_{2}, u_{1}$ and $u_{2}$ denote the degrees of freedom of the finite element functions approaching the solution of the continuous problem, with the same names. The matrices $B_{1}$ and $B_{2}$ are the restriction operators (whose entries are one or zero) corresponding to trace operators of the domains $\Omega_{1}$ and $\Omega_{2}$ along the interface between the two subdomains. The matrices $\widetilde{\mathcal{K}_{1}}$ and $\widetilde{\mathcal{K}_{2}}$ arise from the discretization of the reaction-diffusion subproblems with the interface conditions (2.5)

$$
\widetilde{\mathcal{K}_{j}}=\eta_{j} \mathcal{M}_{j}+\mathcal{K}_{j}+\nu_{i} B_{j}^{T}\left(\mathcal{M}_{\tilde{\beta}_{j}, \Gamma}+\mathcal{K}_{\tilde{\alpha}_{j}, \Gamma}\right) B_{j}, \quad \forall i, j=1,2, i \neq j
$$


Here $\mathcal{K}_{1}$ and $\mathcal{K}_{2}$ are the subdomain stiffness matrices, $\mathcal{M}_{1}$ and $\mathcal{M}_{2}$ are the subdomain mass matrices, $\mathcal{M}_{\Gamma}$ and $\mathcal{M}_{\tilde{\beta}_{j}, \Gamma}$ are the interface mass matrices, and $\mathcal{K}_{\tilde{\alpha}_{j}, \Gamma}$ is the interface stiffness matrix,

$$
\begin{aligned}
\left(\mathcal{M}_{\Gamma}\right)_{i j} & =\int_{0}^{\infty}\left(\phi_{i} \phi_{j}\right)\left(r, \theta_{-}\right) \mathrm{d} r \\
\left(\mathcal{M}_{\tilde{\beta}_{k}, \Gamma}\right)_{i j} & =\int_{0}^{\infty} \tilde{\beta}_{k}(r)\left(\phi_{i} \phi_{j}\right)\left(r, \theta_{-}\right) \mathrm{d} r, \\
\left(\mathcal{K}_{\tilde{\alpha}_{k}, \Gamma}\right)_{i j} & =\int_{0}^{\infty} \frac{\tilde{\alpha}_{k}(r)}{2}\left(\partial_{r} \phi_{i} \partial_{r} \phi_{j}\right)\left(r, \theta_{-}\right) \mathrm{d} r .
\end{aligned}
$$

The functions $\phi_{i}$ and $\phi_{j}$ are the basis functions associated with the degrees of freedom $i$ and $j$ along the interface $\left\{\theta=\theta_{-}\right\}$.

\section{Numerical RESUlts}

The numerical computations with the FreeFem $++{ }^{3}$ software consist in introducing the problem to be solved in each subdomain with its weak formulation. In the weak formulation, the tangential derivative involved in the boundary operator is implemented in FreeFem ++ using the normal vector. In the numerical results we discretize all variational forms by P1-Lagrange finite elements. The effect of singularities associated with corners on domain decomposition methods is transparent when the discretization is fine enough. In order to show such effects without excessively increasing the numerical cost, a refinement of the mesh around the corner is considered. When the domain $\Omega$ is decomposed into two subdomains, one of them must be nonconvex. Assume $x\left(\Omega_{1}\right)=\frac{\theta_{+}-\theta_{-}}{\pi}<1$ and $x\left(\Omega_{2}\right)=\frac{\theta_{-}+2 \pi-\theta_{+}}{\pi}>1$. The first strategy can be applied in $\Omega_{1}$ with the optimal choice given by (4.2), while choosing the coefficient $\alpha_{2}$ to be zero or very small in $\Omega_{2}$ pushes the first artificial singularity as far as possible (second strategy). With this choice, the expansion of the artificial singularities around $r=0$ has the order $O\left(r^{\min \left(t_{2}\left(\Omega_{1}\right), t_{1}\left(\Omega_{2}\right)\right)}\right)$. In our comparison of numerical methods, we shall use the terminology:

1. ICCC for the interface conditions with constant coefficients $\left(\alpha_{\mathrm{opt}}, \beta_{\mathrm{opt}}\right)$ up to the corner.

2. $\mathbf{O C C}$ for the new interface conditions with optimized coefficients at the corner $\left(\alpha_{1,2}, \beta_{1,2}\right)$.

The simple domain which will be considered in our computation is a disc of radius one, centered at the origin and we take a Dirichlet boundary condition $u=0$ on $\partial \Omega$. This global domain is divided into two subdomains $\Omega_{1}$ is a quarter of a disc and $\Omega_{2}=\Omega \backslash \Omega_{1}$, see Figure 2 .

The right-hand side $f$ is taken constant and equal to 1 in the whole domain $\Omega$. We are interesting to the corner singularities, writing our problem in polar coordinates and taking the principal part (Kondratiev theory) the two parameters $\eta_{1}$ and $\eta_{2}$ are suppressed when $r \rightarrow 0$, so in the algorithm we take $\eta_{1}=\eta_{2}=1$. The convergence will be tested, and the logarithmic convergence curve will be plotted with the norm

$$
|e|_{1}=\left(\int_{\Omega}|\nabla e(x)|^{2} \mathrm{~d} x\right)^{1 / 2} .
$$

A comparison of the $L^{\infty}$-norm

$$
\|e\|_{L^{\infty}(\Omega)}
$$

of the error $\left|e_{1}(x)\right|$ will be presented for $\mathbf{O C C}$ and ICCC. Practically, the error function $e^{n}$ at step $n$ is computed as $u^{N}-u^{n}$ where $u^{n}$ is the result after $n$ iterations of the domain decomposition algorithm and $N$ is large enough so that $u^{N}$ is much closer to the (discrete) solution $u^{\infty}$ than the numerical tolerance $\varepsilon=10^{-12}$. Moreover, OCC interface conditions are used to get $u^{N}$. The $u^{N}$ used to compute the error and the solution obtained with a monolithic discretization of the disc are the same. Our stopping criterium is not based

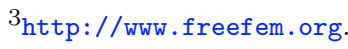




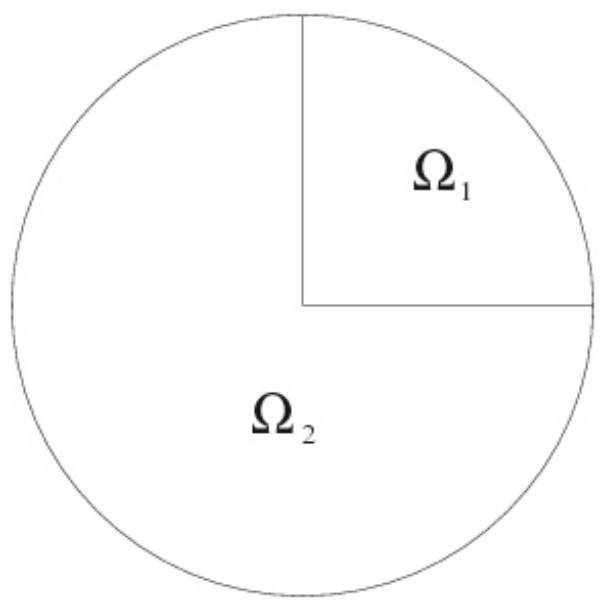

Figure 2. A disc decomposed into two sectors. $\nu(x)=\nu_{1}$ in $\Omega_{1}$ and $\nu(x)=\nu_{2}$ in $\Omega_{2}$ with $\nu_{1} \ll \nu_{2}$.

TABLE 1. Refined mesh around the corner. Number of iterations for different values of $\nu_{2}$ with $\left|e_{1}^{n}\right|_{1}<10^{-6}$.

\begin{tabular}{|c|c|c|c|c|c|c|c|c|c|c|}
\hline$\nu_{2}$ & 2 & 4 & 6 & 8 & 10 & $10^{2}$ & $10^{3}$ & $10^{4}$ & $10^{5}$ & $10^{6}$ \\
\hline Number of iterations with OCC & 12 & 10 & 8 & 8 & 7 & 7 & 3 & 3 & 2 & 1 \\
\hline Number of iterations with ICCC & 22 & 14 & 11 & 9 & 8 & 7 & 3 & 3 & 2 & 1 \\
\hline
\end{tabular}

on the residual norm but on maximum norm of the difference between the discrete solution and its approximation by the iterative algorithm. Thus, there is no bias when we compare interface conditions. We will show by a series of numerical results that the relation (4.2) is optimal, and we limit our study to the geometrical configuration with only one interior corner, corresponding to the case of a circular domain decomposed into two subdomains. Here the disc is decomposed into two sectors with angles $\frac{\pi}{2}$ and $\frac{3 \pi}{2}$. In the nonconvex subdomain $\Omega_{2}$ with angle $\frac{3 \pi}{2}$, the choice of $\alpha_{2}=0$ is done by taking $\widetilde{\alpha}_{2}(r)$ proportional to $r^{2}$ on five elements of the grid close to the corner.

In the convex subdomain $\Omega_{1}$ with angle $\frac{\pi}{2}$, we take $\alpha_{1}=-\frac{2}{\tau_{1}} \frac{1}{\tan \left(\frac{3 \pi}{4} \tau_{1}\right)}$, and we checked that the matching parameter $\left(\frac{\alpha_{\mathrm{opt}}}{\alpha_{1,2}}\right)$ in (2.6) is larger than four times the mesh size $h$ of the refined mesh. We recall that the case $\nu_{1}=\nu_{2}$ was presented numerically in Section 4.3 of [3]. Here we limit our study to the case $\nu_{1} \neq \nu_{2}$. We use a uniform grid everywhere except at the corner $r=0$. The domain decomposition algorithm is tested with various ratios $\frac{\nu_{2}}{\nu_{1}}$ in order to check that (4.2) is optimal (see Tab. 1). We recall that the shorthand OCC for the optimal parameter $\alpha_{1}$ will be compared with the ICCC case (constant coefficients along the interface). If we take $\frac{\nu_{2}}{\nu_{1}}=2$, case where $\nu_{1}=1$ and $\nu_{2}=2$, then with ICCC, 22 iterations are necessary in order to reach $\left|e_{1}^{n}\right|_{1} \leq 10^{-6}$ instead of 12 with OCC. The plot of $\log _{10}\left|e_{1}^{n}\right|_{1}$ with respect to $n$ (see Fig. 3 ) shows the improvement brought by OCC. Table 2 gives the $L^{\infty}$-norm of the error $\left|e_{1}(x)\right|$ with respect to $n$ and shows the improvement given by OCC. The comparison is made between the speed at which the iterates approach an iterate of the $\mathbf{O C C}$ algorithm. This is particularly visible from Table 2: the errors associated to the two interface conditions are comparable for the first 6-7 iterations, then the $\mathbf{O C C}$ conditions outperform the $\mathbf{I C C C}$ ones.

In [4], using optimized second order conditions, a convergence factor of the form $1-O\left(h^{\frac{1}{4}}\right)$ is obtained, and the numerical experiment therein show that the convergence improves as the jump in the coefficient increases. The same property holds for optimized two-sided Robin conditions, and in that case we would gain the additional 
TABLE 2. The case $\nu_{1}=1, \nu_{2}=2$. Comparison of $\left|e_{1}^{n}\right|_{\infty}$ near the corner with $\mathbf{O C C}$ and ICCC with respect to the number of iterations.

\begin{tabular}{|c|c|c|c|c|c|c|}
\hline Iteration & 2 & 4 & 6 & 8 & 10 & 12 \\
\hline OCC & $8.52549 \mathrm{e}-3$ & $5.44888 \mathrm{e}-4$ & $7.09332 \mathrm{e}-05$ & $1.19646 \mathrm{e}-05$ & $2.61246 \mathrm{e}-06$ & $6.51708 \mathrm{e}-07$ \\
\hline ICCC & $8.54753 \mathrm{e}-3$ & $4.38909 \mathrm{e}-4$ & $7.59511 \mathrm{e}-05$ & $2.78231 \mathrm{e}-05$ & $1.43401 \mathrm{e}-05$ & $8.25715 \mathrm{e}-06$ \\
\hline$\frac{\left|e_{1}^{n}\right|_{\infty}, \mathbf{I C C C}}{\left|e_{1}^{n}\right|_{\infty} \text { OCC }} \simeq$ & 1.002 & 0.80 & 1.07 & 2.33 & 5.49 & 12.67 \\
\hline
\end{tabular}

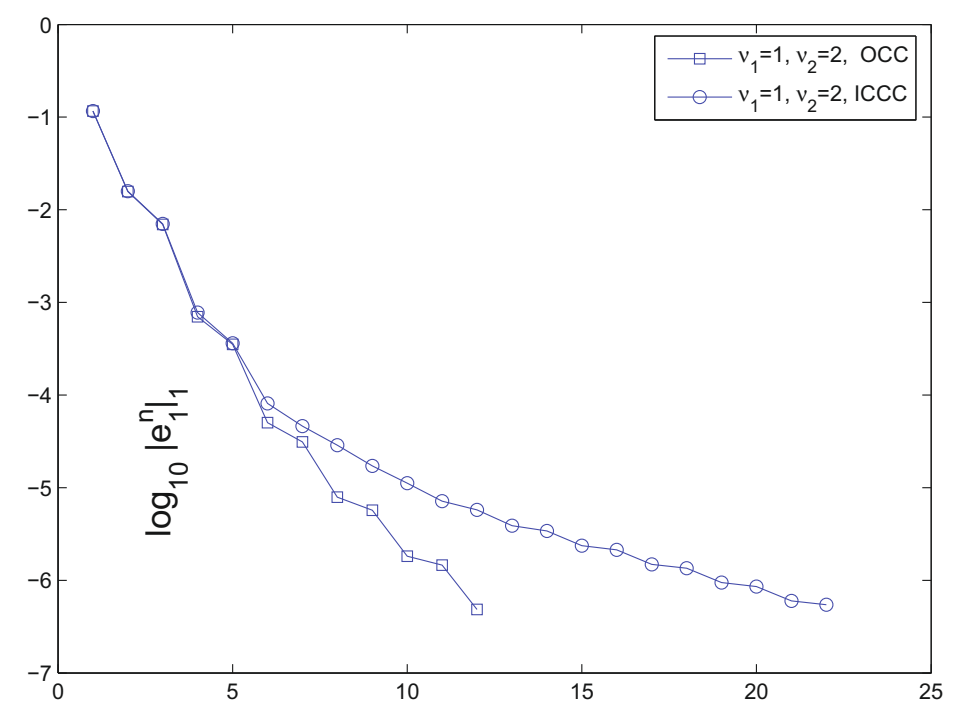

FiguRE $3 . \log _{10}\left|e_{1}^{n}\right|_{1}$ with respect to the number of iterations. The OCC method is represented by the squares, and the ICCC method is represented by the circles, with $\left|e_{1}^{n}\right|_{1}<10^{-6}$, $\nu_{1}=1$ and $\nu_{2}=2$.

advantage that the convergence is independent of the mesh size (see [4]); however for the analysis near the corners, it is more convenient to use second order conditions.

\section{Conclusion}

All the numerical experiments show that the implementation of OCC method improves the behavior of the error in the domain decomposition algorithms. The optimal coefficients for the corresponding transmission conditions are characterized in order to reduce the singularity in the error term. Considering the results obtained in this paper, we plan in the future to tackle the following open questions:

- The numerical results show the robustness of the algorithm with respect to the jumps in the coefficients, but its convergence is still an open problem.

- What happens if the pole of the resolvent is of higher order?

- A good application of our methods in such situations can be observed in two-dimensional domains with more than three subdomains sharing an interior point.

- Extension of our results to the three-dimensional case. 


\section{REFERENCES}

[1] C. Chniti, F. Nataf and F. Nier, Improved interface conditions for the domain decomposition of a non-convex polygonal domain. C. R. Acad. Sci. 342 (2006) 883-886.

[2] C. Chniti, F. Nataf and F. Nier, Improved interface conditions for 2D domain decomposition with corners: a theoretical determination. CALCOLO 45 (2008) 111-147.

[3] C. Chniti, F. Nataf and F. Nier, Improved interface conditions for 2D domain decomposition with corners: Numerical applications. J. Sci. Comput. 38 (2009) 207-228.

[4] O. Dubois, Optimized Schwarz Methods for the Advection-Diffusion Equation and for Problems with Discontinuous Coeffcients. Ph.D. Thesis, McGill University, Montréal (2007).

[5] M.J. Gander, Optimized schwarz methods. SIAM J. Numer. Anal. 44 (2006) 699-731.

[6] P. Grisvard, Singularities in boundary value problems, Research Notes in Applied Mathematics, RMA 22. Springer-Verlag (1992).

[7] C. Japhet and F. Nataf, The Best Interface Conditions for Domain Decomposition Methods: Absorbing Boundary Conditions, in Absorbing Boundaries and Layers, Domain Decomposition Methods - Applications to Large Scale Computation, L. Tourrette and L. Halpern Eds., Nova Science Publishers, Publ. Science (2001) 348-373.

[8] B.N. Khoromskij and G. Wittum, Numerical Solution of Elliptic Differential Equations by Reduction to the Interface, Lect. Notes Comput. Sci. Eng. 36. Springer-Verlag, Berlin (2004).

[9] V.A. Kondratiev, Boundary problems for elliptic equations in domains with conical or angular points. Trudy Moskov. Mat. Obshch. 16 (1967) 227-313.

[10] P.L. Lions, On the Schwarz Alternating Method III: A variant for Nonoverlapping Subdomains, in Third Internationnal Symposium on Domain Decomposition Methods for Partial Differentiel Equations, held in Houston, Texas, March 20-22, Philadelphia, SIAM (1989) 202-223.

[11] F. Nier, Remarques sur les algorithmes de décomposition de domaines, in Séminaire EDP-École Polytechnique (1998-1999). 